Referencia para citar este artículo: Nava-Preciado, J. M. \& Ureña-Pajarito, J. H. (2017). Dominios y valoraciones sobre la felicidad en adolescentes de Guadalajara - México. Revista Latinoamericana de Ciencias Sociales, Niñez y Juventud, 15(1), pp. 443-454.

\title{
Dominios y valoraciones sobre la felicidad en adolescentes de Guadalajara-México*
}

\author{
José MaRía NAVA-PRECIADo** \\ Profesor Universidad de Guadalajara, México. \\ Jesús Heriberto UREÑa-PaJARITo*** \\ Profesor Universidad de Guadalajara, México.
}

\section{Artículo recibido en marzo 7 de 2016; artículo aceptado en junio 10 de 2016 (Eds.)}

- Resumen (analítico): En el presente trabajo, abordamos las valoraciones de un grupo de sujetos adolescentes urbanos sobre lo que significa ser feliz en los micro-contextos de su vida cotidiana. Analizamos los dominios semánticos como resultado de la técnica listas libres, que es una modalidad de la entrevista estructurada; por esta razón, no es representativo en términos estadísticos. Se entrevistaron 264 jóvenes en la ciudad de Guadalajara, en México. Los resultados más relevantes señalan que los adolescentes identifican la felicidad con dominios relacionados con atributos personales, entre los que la salud es el más importante. Además, la vida feliz tiene como cimientos los afectos, principalmente de la familia, los amigos y las amigas. A pesar de su corta edad, estas personas jóvenes evalúan la felicidad con dominios suficientemente valiosos para cultivar una vida dichosa y duradera.

Palabras clave: Adolescencia, familia, salud, afectividad (Tesauro de Ciencias Sociales de la Unesco). Felicidad (Tesauro Open).

\section{Domains and assessments of happiness among adolescents from Guadalajara-Mexico.}

- Abstract (analytical): The present study deals with the valuations that urban adolescents place on what it means to be happy or feel joyful in the micro contexts of their daily lives. The study analyzes semantic domains using the Freelisting technique, which is a form of structured interview. For this reason is not representative in statistical terms. The research was conducted in the city of Guadalajara, Mexico with the participation of 264 young people. The most relevant results show that adolescents identify happiness with domains related to personal attributes, with health being the most important. In addition, the young people stated that a happy life has affection as its foundation, mainly from family and friends. Despite their young age, the participants evaluated happiness with enough significant domains to cultivate a joyful and long lasting life.

\footnotetext{
* En este artículo de investigación científica y tecnológica presentamos una síntesis de los resultados de una investigación realizada entre el 15 de noviembre de 2013 y el 15 de junio de 2015, de la investigación "La percepción de la felicidad en los adolescentes: valoraciones desde el tener, el ser y el representar", dentro de la línea de investigación: "Adolescentes: mundo y vida" que se desarrolla dentro del Cuerpo Académico Epistemología y Axiología, auspiciada por el Departamento de Filosofía de la Universidad de Guadalajara. Registro oficial de la Universidad de Guadalajara, Centro Universitario de Ciencias Sociales y Humanidades (Coordinación de Investigaciones) del 6 de octubre de 2014. Área de conocimiento: Otrags Humanidades, sub-área filosofía.

** Doctor en educación. Profesor titular "C" del Departamento de Filosofía, Universidad de Guadalajara, miembro del SNI nivel I y responsable del Cuerpo Académico Epistemología y Axiología. Correo electrónico: jnava_preciado@yahoo.com.mx

*** Maestro en filosofía. Profesor investigador titular “A” miembro del Cuerpo Académico Epistemología y Axiología y responsable de la línea de investigación: Problemas epistemológicos y éticos. Correo electrónico: maloso36@gmail.com
} 
Key words: Adolescence, family, health, affectivity (Unesco Social Science Thesaurus). Happiness (Open Thesaurus).

\section{Domínios e valorações sobre a felicidade em adolescentes de Guadalajara, no México}

- Resumo (analítico): O presente trabalho aborda as valorações de um grupo de adolescentes urbanos sobre o que significa ser feliz nos microcontextos da vida cotidiana. O estudo analisa os domínios semânticos como resultado da técnica listas livres, uma modalidade da entrevista estruturada, por esta razão não é representativo em termos estatísticos. Foram entrevistados 264 jovens da cidade de Guadalajara, no México. Os resultados mais relevantes arrojam que os adolescentes identificam a felicidade com domínios relacionados a atributos pessoais, sendo a saúde o mais importante; além disso, a vida feliz tem como base os afetos, principalmente da família e dos amigos. Apesar da sua curta idade estes jovens avaliam a felicidade com domínios suficientemente valiosos como para cultivar uma vida longa e ditosa.

Palavras-chave: Jovem, avaliação, família, saúde, afetividade (Thesaurus de Ciências Sociais da Unesco). Felicidade (Thesaurus Open).

-1. Introducción. -2. Los criterios metodológicos. -3. Resultados. -4. Discusión. -5. Conclusiones. -Lista de referencias.

\section{Introducción}

El ser humano históricamente se ha cuestionado si es feliz o infeliz; se ha preguntado sobre las razones que posee para calificar una vida de felicidad; ha indagado sobre los criterios que le permiten determinar en qué medida puede serlo. Ya en la antigüedad vemos plasmadas reflexiones en torno a esta temática. De manera general, los filósofos asociaban la felicidad al modus vivendi de las personas. Según Sócrates, los seres humanos deseamos siempre lo bueno, siendo la posesión de los bienes la que conduce a la felicidad: "Habíamos convenido -recordéque si poseyésemos muchos bienes seríamos felices y dichosos" (Platón, 271a-307c - trad. en 1983 , p. 280b). Si bien es cierto que todos los seres humanos deseamos ser felices con dicha posesión, como lo advierte Sócrates, no se refiere a bienes externos como la riqueza, sino a los bienes interiores del hombre, es decir, a la virtud y la sabiduría. Así, la persona logra ser feliz cuando recurre a la reflexión, al saber y a la bondad. En ese mismo sentido, para Platón la verdadera felicidad consistía en un estado mediante el cual el alma accede al encuentro con Dios, asociándola a un modo de vida donde el alma es prioritaria. Si ésta concentra las actividades superiores del intelecto, entonces la vida feliz es la que se dedica a la contemplación
(Brisson, 2011); mientras que para Aristóteles la felicidad es el más excelso de los bienes en el orden de la actividad humana; el estagirita llegó a considerarla como un fin en sí mismo.

Así, una de las posibles formas de evaluar una vida como feliz es estar satisfecho con lo logrado a lo largo de la existencia, misma que se compone de expectativas y satisfacciones, así como de la evaluación que hagamos de nuestras propias actuaciones. De esta manera, un sujeto adolescente puede evaluar cómo le ha ido en la vida, y partiendo de ello nos podría decir qué tan feliz es; esto es, el sentimiento de felicidad de un agente no se puede medir en momentos, dado su carácter fugaz. Por el contrario, determinar si una existencia es feliz implica que se evalúa después de hacer un balance de todas las cosas que ha tenido y disfrutado. Así, hablamos en más de un sentido de que su vida tiene algún tipo de significado (Singer, 1995). Además, como sostiene Aristóteles (trad. en 2012), la felicidad exige un suplemento de prosperidad que sólo puede medirse vitalmente. Como se observa, en la antigüedad los filósofos discutieron ampliamente este tema (Salazar \& Arenas, 2016), y algunas de sus cavilaciones éticas siguen siendo relevantes en la actualidad tanto para el debate como para la vida cotidiana (Rojas, 2013). Sin embargo, hoy en día los principales estudios sobre la felicidad 
no se dan necesariamente en la filosofía. Fundamentalmente, las investigaciones se pueden enmarcar en dos grandes discusiones: por un lado, se tienen los trabajos que abordan el tema desde la psicología cognitiva y, por el otro, se encuentran los estudios que correlacionan las cuentas nacionales del bienestar subjetivo con los niveles de felicidad de la sociedad (Diener, 2013). Estos últimos trabajos quizá son los más conocidos, debido a que recientemente algunos países ${ }^{1}$ aplicaron encuestas a amplios sectores de la población con la finalidad de comparar los índices de bienestar a través de un índice de felicidad (Loewe, 2011). Como nos damos cuenta, la narrativa de la felicidad es fundamental; por eso cobra sentido preguntarse qué tan felices somos y cuáles son los dominios que abonan a su conquista. En el marco de estos acercamientos teóricos y empíricos llevamos a cabo la presente investigación, teniendo como propósito central reflexionar el tema con las gentes adolescentes. Es decir, buscamos hacer este trabajo encontrando una manera práctica de acercar la discusión con estos interlocutores e interlocutoras, con la intención de conocer más sus intereses y de explorar ciertas valoraciones acerca de su mundo, porque se encuentran en una edad que abiertamente presenta desafíos en la configuración de un proyecto de vida.

En este escrito, los referentes teóricos son las reflexiones que los grandes filósofos han expresado sobre el tema. Sabemos que lo han escudriñado con seriedad y profundidad, pero también se debe aceptar que no hay homogeneidad de criterio entre ellos. Sin embargo, a pesar de lo diverso y heterogéneo de sus teorías, se puede reconocer un pensamiento en común que sirve de punto de partida. Por esta razón, de antemano se descarta inclinarnos hacia un planteamiento filosófico particular, pues recuperamos lo conveniente de algunos de ellos para orientar y guiar la investigación. Desde luego que la felicidad es un tema complejo, como dice Marías (1989), porque tiene que ver con realidades que no son cosas $\mathrm{y}$, al mismo tiempo, son elusivas; por ello se

1 En México, la Primera Encuesta Nacional sobre el Bienestar se realizó en el año 2012 por parte del Instituto Nacional de Estadística y Geografía (Inegi) y los resultados fueron publicados en el mes de noviembre de ese año. podría cuestionar la posibilidad de llevar a cabo un estudio de este tipo. Sin embargo, como el mismo Marías sostiene, se debe buscar e indagar sobre el camino hacia la felicidad ya que nos es inherente como seres humanos. Además, es pertinente este tipo de estudios porque permiten conocer a qué se le da valor cuando se piensa en la felicidad (Rodríguez, 2015). Así pues, las preguntas de investigación son las siguientes: ¿Con qué dominios semánticos identifican los sujetos adolescentes la felicidad al poner en juego las valoraciones del tener, el ser y el representar? Para las personas adolescentes, ¿cuáles dominios son más relevantes para un proyecto de vida feliz?

\section{Los criterios metodológicos}

La investigación toma como eje central las valoraciones que los individuos adolescentes tienen sobre lo que significa ser feliz, o sentirse dichoso, en los micro-contextos de su vida cotidiana, los cuales están rodeados de muchas necesidades materiales creadas por la sociedad a la que pertenecen. El estudio, al privilegiar los dominios semánticos a través de listas libres, no se propone ser representativo en términos estadísticos; por sus objetivos se enmarca en un enfoque interpretativo debido a que las listas y las razones argumentativas de los agentes interlocutores, sobre la elección y los consensos de los dominios, se dotan de significados y se insertan en el contexto de las deliberaciones que se realizan acerca de la felicidad desde nuestro propio marco de comprensión. En este tipo de enfoques "El investigador hace preguntas más abiertas, recaba datos expresados a través del lenguaje escrito, verbal y no verbal, así como visual, los cuales describe, analiza y los convierte en temas que vincula y reconoce sus tendencias personales" (Hernández, Fernández \& Baptista, 2014, p. 8). El supuesto básico del que parte el presente trabajo radica en afirmar que las valoraciones de las adolescentes $y$ los adolescentes acerca de la felicidad están relacionados con las formas de sentirse dichosos y de cómo les va en y con el mundo. Debemos reconocer, como dice Aristóteles (trad. en 2012), que la gente joven, por su propia naturaleza, no tiene experiencia suficiente en las acciones 
de la vida; esto significa que las valoraciones de los entrevistados se ven limitadas por este sabio criterio aristotélico. Por tanto, lo anterior es una condición que no se deja de lado en este artículo.

Población objetivo. Realizamos la investigación con una muestra homogénea ${ }^{2}$ de estudiantes del primer semestre de seis escuelas preparatorias de la Zona Metropolitana de Guadalajara (tabla 1), tomando como criterio que estuvieran representados los distintos subsistemas que ofrecen servicios educativos, y que por su propia estructura y organización cuentan con planes de estudios diferenciados. La edad promedio de los estudiantes y las estudiantes oscila entre 15 y 16 años. Las razones por las cuales hicimos el estudio con personas de esta edad, son las siguientes: (1) en este período se observan importantes cambios actitudinales y éticos que determinan su visión de la realidad; (2) se encuentran en la plenitud de las adolescencias; (3) lo que se cultive en estos años es determinante para el logro de un plan de vida con futuro pleno; (4) están en la búsqueda de modelos éticos con los cuales identificarse. Los interlocutores e interlocutoras de estas edades son quienes aportan información relevante para los fines del estudio; por esta razón el muestreo tiene un carácter intencional, estrategia propia del paradigma cualitativo (Yuni \& Urbano, 2014).

Tabla 1. Subsistemas educativos y escuelas preparatorias donde realizamos el estudio.

\begin{tabular}{|c|c|c|c|}
\hline Subsistema educativo & Tipo de servicio & $\begin{array}{l}\mathrm{N}^{\circ} \text { de estudiantes } \\
\text { por grupo }\end{array}$ & $\begin{array}{c}\text { Conformación del } \\
\text { grupo }\end{array}$ \\
\hline $\begin{array}{l}\text { Colegio de Bachilleres del } \\
\text { Estado de Jalisco (Cobaej) }\end{array}$ & Público & 46 & Mixto \\
\hline $\begin{array}{l}\text { Colegio de Estudios } \\
\text { Científicos y Tecnológicos del } \\
\text { Estado de Jalisco (Cecytej) }\end{array}$ & Público & 38 & Mixto \\
\hline $\begin{array}{l}\text { Colegio Nacional de } \\
\text { Educación Profesional Técnica } \\
\text { (Conalep) }\end{array}$ & Público & 39 & Mixto \\
\hline $\begin{array}{l}\text { Sistema de Educación Media } \\
\text { Superior Universidad de } \\
\text { Guadalajara (Sems) }\end{array}$ & Público & 42 & Mixto \\
\hline $\begin{array}{l}\text { Universidad del Valle de } \\
\text { Atemajac (Univa) }\end{array}$ & Privado & 50 & Mixto \\
\hline Albergue "Villa de los Niños" & $\begin{array}{l}\text { Asociación Civil } \\
\text { (A. C.) }\end{array}$ & 49 & Hombres \\
\hline \multicolumn{2}{|l|}{ Total } & 264 & \\
\hline
\end{tabular}

Técnica. Concluimos que el listado libre era la técnica más pertinente para los objetivos del estudio. Listas libres es una técnica que pertenece propiamente a la antropología cognitiva (Romney, Weller \& Batchelder, 1986), siendo una modalidad de la entrevista estructurada, porque plantea una serie de preguntas a los sujetos informantes sobre un dominio específico con la finalidad de encontrar consensos que

En la muestra homogénea se busca que los sujetos investigados tengan el mismo perfil o las mismas características. se manifiestan por el nivel de relevancia y el orden que le atribuyen a cada listado. Con base en esa técnica y nuestras reflexiones teóricas, la serie de preguntas giró sobre tres ejes que dan cuenta del tema de la felicidad: (1) lo que la persona tiene: dinero, trabajo, familia, amigos; (2) lo que la persona es: salud, belleza, inteligencia, jovialidad; (3) lo que la persona representa: fama, éxito, aceptación de los demás. En cada respuesta les pedimos a los sujetos investigados que profundizaran 
en las razones de su elección, para contar con mayor información. Codificamos las respuestas a través del paquete Anthropac.

\section{Resultados}

La felicidad y sus dominios con los atributos de la persona. El sentimiento hacia sí mismo es fundamental para cualificar qué tanto se es feliz o desdichado. El sentirse feliz se relaciona con lo que uno mismo representa para sí. El listado que los individuos entrevistados mencionan con mayor relevancia para ser felices es estar sano (tabla 2). Sócrates decía que estar sano es una dicha. Tal idea se encuentra presente también en Schopenhauer (2008, p. 55) quien considera que la salud es el mayor de los bienes: "Con ella todo se convierte en manantial de placer; sin ella, por el contrario, no podríamos disfrutar de un bien exterior, de cualquier naturaleza que sea". En realidad, cuando una persona está sana tiene lo fundamental para sentirse dichosa. Al analizar las respuestas de nuestros adolescentes encontramos que la mención estar sano presenta un nivel de significancia de .0871, mientras que el índice de prominencia es de 1.65. Entre las expresiones que utilizan los individuos entrevistados para referirse a la relevancia de la lista están las siguientes: "Un cuerpo sano, pues la salud, debemos agradecer por tenerla, es algo muy valioso y si nos ponemos a pensar, de ésta podemos desarrollar nuevas cosas; tener salud es tener capacidad para lograr cualquier cosa, para vivir, para sentir nuevas cosas, pues de ésta dependen las metas, las experiencias, la madurez, podemos lograr lo que queramos, lo demás son excusas. La salud es algo muy valioso y que no todos tenemos" (M); "Cuando estoy enfermo no soy feliz y cuando estoy sano es lo mejor, lo más importante de la vida es la vida misma" $(\mathrm{H})$; "Porque si no estás sano no serías feliz y también porque estar sano es la puerta de todo lo demás" (H); "La salud es la base de la felicidad, una persona sana puede hacer lo que más le gusta de la forma que quiera y cuando quiera" $(\mathrm{M})^{3}$.

3 A lo largo del trabajo utilizamos la $\mathrm{M}$ para referirnos a la narrativa de las mujeres y $\mathrm{H}$ para el caso de los hombres.
Tabla 2. Felicidady dominios relacionados con los atributos de la persona.

\begin{tabular}{|l|c|c|}
\hline $\begin{array}{c}\text { Mención } \\
\text { identificada }\end{array}$ & $\begin{array}{c}\text { Rango de } \\
\text { prominencia }\end{array}$ & $\begin{array}{c}\text { Correlación } \\
\text { sobresaliente* }^{*}\end{array}$ \\
\hline Estar sano o sana & 1.65 & 0.871 \\
\hline Ser alegre & 2.09 & 0.783 \\
\hline Ser inteligente & 3.16 & 0.568 \\
\hline Ser joven & 3.95 & 0.410 \\
\hline Tener buen físico & 4.16 & 0.368 \\
\hline
\end{tabular}

* La correlación sobresaliente indica el lugar de mención en el dominio.

Fuente: Elaboración propia.

La siguiente lista con mayor relevancia para ser feliz es ser alegre; el nivel de mención arroja una significancia de 0.783. Como se observa, es una significancia también muy alta. Sin lugar a dudas, la expresión alude al carácter de los agentes, a sus atributos personales que sirven de herramienta para hacer posible su encuentro con el mundo. Sobre el particular, Nussbaum (2004, p. 432) afirma lo siguiente: "Las excelencias más importantes del carácter residen, por así decirlo, en la persona; son estados de la persona". Lo que Nussbaum sostiene es que el sentirse feliz se relaciona con lo que uno mismo representa para sí; eso queda claramente expresado a continuación: "Si tienes buena actitud toda tu vida será cómoda y mejor comunicada" (M); "La autoestima es el primer paso para estar bien, ser positivos, alegres, divertidos, contentos" $(\mathrm{H})$; "Porque si eres alegre puedes hacer todo de buen modo" (M); "Por medio de la alegría y el lado positivo de todo, es más fácil aceptar las cosas que te matan. Por ello llegará la felicidad" $(\mathrm{H})$; "Porque si eres alegre siempre le ves el lado bueno a las cosas" (M); "Cuando no somos alegres la vida se nos hace aburrida y cuando somos alegres se hace siempre feliz, aunque estemos enfermos" (H).

La felicidad y sus dominios con el tener. Aquí se enfatizan dos significados asociados al tener: a) la posesión de bienes materiales (calzado, alimento y casa, por ejemplo); y b) la posesión de bienes inmateriales, por ejemplo, tener relaciones, una familia, amistades, etc. A1 respecto, la lista que ocupa el primer lugar de mención en la pregunta para ser feliz es tener 
familia (tabla 3). El índice de prominencia es de 1.20 , con un nivel de significancia muy alto. Algunas expresiones ilustrativas son las siguientes: "Porque la familia es la base de todo, te apoya y te va a entender mejor que nadie" (M); "Es más importante tener a las personas que quieres y que jamás te cambiarán por cosas materiales. Es muy importante convivir con la familia" (M); "Es lo más importante, el crecer con los que te ven crecer" $(\mathrm{H})$; "Se me ha sido asignada una familia, la cual gracias a ella es que hoy estoy aquí, vivo y agradecido por cada uno de ellos y por mí mismo" $(\mathrm{H})$.

Tabla 3. Felicidady dominios relacionados con el tener.

\begin{tabular}{|l|c|c|}
\hline \multicolumn{1}{|c|}{$\begin{array}{c}\text { Mención } \\
\text { identificada }\end{array}$} & $\begin{array}{c}\text { Rango de } \\
\text { prominencia }\end{array}$ & $\begin{array}{c}\text { Correlación } \\
\text { sobresaliente }\end{array}$ \\
\hline Tener familia & 1.20 & 0.961 \\
\hline $\begin{array}{l}\text { Tener amigos y } \\
\text { amigas }\end{array}$ & 2.22 & 0.757 \\
\hline Tener dinero & 3.19 & 0.563 \\
\hline Tener propiedades & 3.89 & 0.423 \\
\hline Vivir con lujos & 4.53 & 0.295 \\
\hline
\end{tabular}

Fuente: Elaboración propia.

Por otra parte, también hubo estudiantes que dijeron que tener amigos y amigas es lo más importante: "Los amigos son como tu familia porque saben más de ti" (M); "Porque es con las personas que te diviertes más y les puedes contar todo" $(\mathrm{H})$; "Si no tienes familia así te quedes sin ella los amigos te ayudan" $(\mathrm{H})$; "Ellos siempre están contigo no importa lo que pase te quieren como eres" (M); "Porque ellos te van a apoyar en las buenas y en las malas" (H).

La felicidad y sus dominios sobre lo que la persona representa para los demás. Como seres gregarios, la opinión de los otros también tiene peso al determinar si se es feliz o no. Al respecto, los resultados muestran que en el caso de nuestros interlocutores e interlocutoras encontramos que ser reconocido por los amigos $y$ amigas es valorado como muy importante; el índice de prominencia es de 1.38 con un nivel de significancia de 0.924; ninguna otra mención tiene este peso (tabla 4). Tal relevancia se manifiesta por medio de los siguientes enunciados: "Con ellos se convive mucho y es bueno saber que les importas" (M); "Es bueno que tus amigos conozcan de lo que eres capaz" (H); "Eso te va a dar seguridad de ti mismo, cuando tienes amigos que te reconocen" $(\mathrm{M})$;" Es importante ser valorado por las virtudes $\mathrm{y}$ capacidades que tenemos" $(\mathrm{H})$; "Creo que te da gusto que un amigo te reconozca por ser alguien bueno" (M); "Cuando hacemos algo bueno, los amigos siempre nos admiran y quieren seguir tu ejemplo y te sientes feliz" $(\mathrm{H})$.

Tabla 4. Felicidady dominios relacionados sobre lo que la persona representa para los demás.

\begin{tabular}{|l|c|c|}
\hline \multicolumn{1}{|c|}{$\begin{array}{c}\text { Mención } \\
\text { identificada }\end{array}$} & $\begin{array}{c}\text { Rango de } \\
\text { prominencia }\end{array}$ & $\begin{array}{c}\text { Correlación } \\
\text { sobresaliente }\end{array}$ \\
\hline $\begin{array}{l}\text { Ser reconocido } \\
\text { por los amigos y } \\
\text { amigas }\end{array}$ & 1.38 & 0.924 \\
\hline Recibir aplausos & 2.41 & 0.718 \\
\hline Tener fama & 3.04 & 0.591 \\
\hline $\begin{array}{l}\text { Tener poder sobe } \\
\text { los demás }\end{array}$ & 4.08 & 0.385 \\
\hline $\begin{array}{l}\text { Tener cargos } \\
\text { políticos }\end{array}$ & 4.10 & 0.380 \\
\hline
\end{tabular}

Fuente: Elaboración propia.

En un menor porcentaje, hubo estudiantes que basan la felicidad en menciones como ser famoso o famosa y recibir aplausos que, en sentido estricto, pudieran ser expresiones que se refieren a lo mismo: se busca que los demás nos reconozcan. Los comentarios sobre ser famoso o famosa giran en alocuciones como: "Sería reconocida por todos, significa que sería importante y cambiar algo" (M); "A las personas nos gusta ser reconocida por todos" (H); "Ser famoso hoy en día es algo grande es como ser popular donde sea" $(\mathrm{H})$. En cuanto a recibir aplausos algunas expresiones ilustrativas se escriben a continuación: "Pues no sé, señal de que estoy haciendo bien las cosas XD" (M); "Recibir aplausos me motiva para hacer las cosas" $(\mathrm{H})$; “Te sentirás bien por lo que has hecho o dicho al igual cuando ayudas a una persona le das las gracias" (M). 
La felicidad y la infelicidad en el yo proyectivo de los adolescentes. Marías (1989, p. 20) afirma que la: "Felicidad es mi felicidad, no solo en el sentido de que tiene que poseer un carácter individual, particular, diferenciado, sino sobre todo en que tiene que tener conexión con el quién proyectivo que es cada uno de nosotros." La pertinencia de esta cita, pensando en que había que particularizar más las preguntas para referirlas a ellos mismos, llevó a cuestionarlos sobre qué los hace felices o infelices. Así, desde su yo proyectivo lo que más los hace felices es el hecho de tener una familia. El nivel de mención arroja una significancia de 0.926, seguido de la mención estar sano que, a su vez, presenta un nivel de 0.763 (tabla 5). Sobre la mención en este dominio se agrupan expresiones como las siguientes: "La familia te apoya en las buenas y en las malas y nunca te dejarán solo y pues, tener familia es un gran privilegio" (M); "Es la raíz de la vida" $(\mathrm{H})$; "Porque la familia te ama y te dan lo que necesitas" (M); "Al momento de convivir con mi familia siento el apoyo y aprecio de todos lo que me hacen estar muy feliz" $(\mathrm{H})$; "Para mí son lo más importante, son el único apoyo que tengo y gracias a ellos disfruto lo bueno de la vida, soy feliz y aprendo de su ejemplo" (M); "Mi familia me ha enseñado al paso de los años la vida de la mejor manera, aceptando mis aciertos y aceptando mis errores, y aunque vivir no siempre es estar feliz, siempre hay un motivo para poder levantarse" (M).

En relación con la mención estar sano se encuentran expresiones como las siguientes: "Si no estás sano no puedes disfrutar de nada ni de tu familia o de tus amigos menos" (M); "Si no estuviera sano me impediría todo lo demás porque si tuviera alguna enfermedad no iría a la escuela, no tendría amigos, no disfrutaría la vida" $(\mathrm{H})$; "Porque es lo importante para poder hacer todo lo que quiera y poder ser feliz" (M); "Si no estás sano ni tú ni la familia son felices, la familia por tu dolor o enfermedad y tú porque ves a tu familia triste por tu enfermedad" $(\mathrm{H})$; "Hago lo que me gusta, estoy feliz y de buen humor y no me siento agotado" $(\mathrm{H})$.
Tabla 5. Dominios que hacen fel es a los individuos adolescentes.

\begin{tabular}{|l|c|c|}
\hline \multicolumn{1}{|c|}{$\begin{array}{c}\text { Mención } \\
\text { identificada }\end{array}$} & $\begin{array}{c}\text { Rango de } \\
\text { prominencia }\end{array}$ & $\begin{array}{c}\text { Correlación } \\
\text { sobresaliente }\end{array}$ \\
\hline Tener familia & 1.74 & 0.926 \\
\hline Estar sano o sana & 3.37 & 0.763 \\
\hline $\begin{array}{l}\text { Convivir con } \\
\text { amigos y amigas }\end{array}$ & 4.28 & 0.672 \\
\hline $\begin{array}{l}\text { Disfrutar lo } \\
\text { bueno de la vida }\end{array}$ & 5.00 & 0.600 \\
\hline $\begin{array}{l}\text { Poder ir a la } \\
\text { escuela }\end{array}$ & 5.09 & 0.591 \\
\hline $\begin{array}{l}\text { Sacar buenas } \\
\text { calificaciones }\end{array}$ & 5.65 & 0.535 \\
\hline $\begin{array}{l}\text { Ser apreciado por } \\
\text { mis compañeros } \\
\text { y compañeras }\end{array}$ & 6.37 & 0.463 \\
\hline $\begin{array}{l}\text { Tener tiempo } \\
\text { libre }\end{array}$ & 7.28 & 0.372 \\
\hline Tener buen físico & 7.81 & 0.258 \\
\hline $\begin{array}{l}\text { Tener dinero para } \\
\text { comprar lo que } \\
\text { quiero }\end{array}$ & 8.42 & 0.319 \\
\hline
\end{tabular}

Fuente: Elaboración propia.

Los estudiantes y las estudiantes identifican la infelicidad, principalmente, con las menciones no tener familia y estar enfermo $o$ enferma. En el primer caso se encuentra un índice de significancia de 0.836 , con una medida de prominencia de 2.64 , mientras que en el segundo se observa una significancia de 0.693 con un índice de prominencia de 4.07 (tabla 6). Entre algunas expresiones que permiten ejemplificar el primer dominio resaltan las siguientes: "Necesito de ellos o más bien ellos son un complemento en mi vida que me ayudan a seguir adelante" (M); "Caería en depresión y no sabría qué hacer, caería en vicios o en drogas" $(\mathrm{H})$; "Sería una persona triste y amargada, porque no habría nadie a mi lado" $(\mathrm{M})$; "Porque no tendría consejos ni apoyo para poder enfrentar la vida" $(\mathrm{H})$; "Aunque muchas personas no tienen una, yo corrí con la suerte de crecer con una y es lo más importante para mí en la vida" (M); "Sin tu familia, no podrás recibir apoyo, convivir con ellos, y tener su amor" $(\mathrm{H})$ : "Nuestra familia es la base de nuestra vida, el ejemplo, las primeras personas que te brindan amor y felicidad" (M). 
Tabla 6. Dominios que hacen infelices a los individuos adolescentes.

\begin{tabular}{|l|c|c|}
\hline \multicolumn{1}{|c|}{$\begin{array}{c}\text { Mención } \\
\text { identificada }\end{array}$} & $\begin{array}{c}\text { Rango de } \\
\text { prominencia }\end{array}$ & $\begin{array}{c}\text { Correlación } \\
\text { sobresaliente }\end{array}$ \\
\hline No tener familia & 2.64 & 0.836 \\
\hline $\begin{array}{l}\text { Estar enfermo o } \\
\text { enferma }\end{array}$ & 4.07 & 0.693 \\
\hline $\begin{array}{l}\text { No tener amigos } \\
\text { ni amigas }\end{array}$ & 4.27 & 0.673 \\
\hline $\begin{array}{l}\text { Los problemas } \\
\text { entre mis padres }\end{array}$ & 5.02 & 0.528 \\
\hline $\begin{array}{l}\text { Sacar malas } \\
\text { calificaciones }\end{array}$ & 5.61 & 0.539 \\
\hline $\begin{array}{l}\text { No poder ir a la } \\
\text { escuela }\end{array}$ & 5.90 & 0.510 \\
\hline Sufrir bullying & 6.08 & 0.492 \\
\hline No tener dinero & 6.51 & 0.449 \\
\hline $\begin{array}{l}\text { No tener tiempo } \\
\text { libre }\end{array}$ & 6.94 & 0.406 \\
\hline Estar feo o fea & 7.97 & 0.303 \\
\hline
\end{tabular}

Fuente: Elaboración propia.

\section{Discusión}

A partir de las propias experiencias de nuestros sujetos adolescentes, se puede constatar cómo se valora el sentido de la felicidad; observamos que está en relación con las formas de verse, de sentirse y actuar con el mundo, tal como ellos y ellas lo han expresado. Esta primera reflexión lleva a sostener que el cultivo de la felicidad requiere de una tenaz persistencia para edificarla sobre bases sólidas a lo largo de sus biografías. De antemano se sabe que es una mezcla de experiencias felicitarias y de momentos de desventura; no puede ser de otra manera. Sin embargo, cada etapa de la vida está marcada por esas experiencias que definen las trayectorias vitales, esto es, el modo de ser feliz. Todos los seres humanos experimentamos la felicidad, algunos quizás más que otros. Esto tiene que ver con una serie de atributos relacionados con el ser de los agentes, de los cuales dos son centrales, según la posición de los individuos entrevistados: la salud y el carácter. En términos generales, no se puede poner en duda el valor de estos dominios por lo que representan en la vida de cualquier persona. Estar sano o sana es un atributo altamente valorado por ellos y ellas; lo reconocen como algo fundamental en la consecución de la felicidad, entre otras razones porque: "La salud es un bien completo y perfecto porque no requiere otro bien para ser objeto del deseo" (Zagal, 2013, p. 86). Esta idea de Zagal tiene una implicación práctica, porque la felicidad transcurre de manera paralela a nuestra salud física y mental. Para encontrarle sentido a la existencia, nuestros adolescentes demandan estar sanos. Esto significa que si lo están, muchas otras acciones y metas en su vida se pueden trazar. La salud es un agradable proceso físico y psíquico que los hace sentirse libres, plenos y alegres. Como los interlocutores e interlocutoras lo expresan, se trata de la puerta a todo lo demás. Pero además, estar sano afecta en su totalidad la vida de las personas, dado que encontrarse libre de cualquier enfermedad, o sanar si se padece, es condición para llevar a cabo cualquier actividad. Es por ello que se puede afirmar que la salud no es un asunto trivial, como se puede pensar, sino que constituye la principal fortaleza de un sujeto adolescente: insertarse en el mundo y trazarse un plan de vida, no sólo para el presente inmediato sino con la mirada puesta en el porvenir, implica estar sano o sana. De este modo, la salud posibilita sentir $\mathrm{y}$ apasionarse por las tareas que cotidianamente le dan sentido a cada momento de su existencia. Estar sano o sana, siguiendo la narrativa de nuestros adolescentes, permite experimentar las cosas del mundo, de las cuales no nos podemos sustraer en el diario vivir porque, precisamente, son ellas las que abonan al proyecto de un mundo feliz. Es así que la salud se convierte en el principal atributo que cualquier ser humano puede tener como algo valioso en sí mismo. Contar con salud, permite compartir nuestra felicidad con los demás y con las cosas del mundo. Un pobre sano es más feliz que un rey enfermo, como bien dice Schopenhauer (2008). Las experiencias felicitarias, o la ausencia de ellas, dejan huella en nuestra biografía y van configurando nuestro carácter, ese peculiar modo de ser y estar con y en el mundo. En ese sentido, es común escuchar expresiones como: tiene buen humor, siempre está contenta, eres pesimista, mismas que cuales refieren precisamente a ese modo de ser. Como lo valoran los estudiantes y las estudiantes, la 
felicidad depende en cierto modo de nuestro carácter; ser alegre es manifestar el optimismo por las experiencias que se disfrutan día tras día. Esto permite afirmar, con cierta propiedad, que el tipo de carácter es una conditio favorable o desfavorable para que las personas adolescentes conquisten la felicidad. En muchas ocasiones ese núcleo subyacente de buen carácter, como arguye Nussbaum (2004), hace ver las cosas con más serenidad y permite estar en condiciones de hacer frente a lo adverso. Ser alegre es vivir las cosas de la vida cotidiana con cierta intensidad, con una mirada de mesura y cordialidad con el mundo; por esta razón se vuelve un aprendizaje del diario vivir con sus atractivos y sinsabores, en algún sentido: "Un soplo de alegría llena de luz una jornada que hasta entonces no era ni buena ni mala, pero que de pronto se tiñe de un color especial" (Bruckner, 2008, p. 117). Es verdad que la alegría anima a no caer en la desesperación cuando las expectativas no coinciden con las vivencias. Dicho en otras palabras: la actitud hacia el mundo nace de un proyecto acompañado de optimismo. Sin embargo, conviene aclarar que la alegría debe ser cultivada en un entorno afectuoso, porque una adolescente o un adolescente no pueden estar sometidos a condiciones familiares o escolares adversas en la formación del buen carácter; éste se construye, sin duda, en un entorno apropiado. La importancia del carácter es tal, que permite, en primera instancia, concebir al mundo en el que se vive. Así, según nuestro parámetro, se puede ver el mundo de determinado color, esto es, se torna aburrido o interesante. El carácter en estos sujetos adolescentes, por tanto, es determinante para ver qué tan agradables o desagradables son sus semejantes y las cosas; eso es primordial porque, como lo postula Schopenhauer (2008), la concepción del mundo reside en nuestro interior. Lo que brinda nuestro carácter es tan fundamental que en muchas ocasiones se llega a identificar que de él depende la felicidad. Cuando se enfrentan con optimismo los sucesos del mundo se hace porque somos felices y viceversa, por esta razón se llega a esgrimir que: “... la felicidad también depende de aquello que podríamos identificar en general como alegría" (Foot, 2002, p. 153). Sin embargo, la felicidad no se puede alcanzar de una sola manera en cualquier adolescente; podrán tener salud, ser de carácter jovial y ser inteligentes, pero estos atributos no son suficientes para que aquella se despliegue. La vida feliz, desde las valoraciones del tener, tiene como cimiento los afectos, siendo éstos centrales para evaluar qué tan dichoso se es. El cariño y la amistad que reciben y dan a los demás determina su vida venturosa: "La felicidad básica depende sobre todo de lo que podríamos llamar un interés amistoso por las personas y las cosas" (Russell, 2003, p. 94). Sin duda, y la experiencia mundana lo dice, en cualquier caso nuestro interés por las personas se centra fundamentalmente en el afecto. El sentir afecto de los demás y corresponder a él se traduce en un ingrediente de felicidad. Como se constata, nada hay tan grato para un individuo adolescente como el amor de la familia y la amistad de los amigos y amigas; cuando se experimentan esos lazos afectivos se disfruta de la vida, pero además por el placer que despiertan dignifican y fortalecen su carácter. ¿Qué esperan los sujetos adolescentes de la familia? Para ellos la familia tiene una doble valoración: como algo especial en el presente que protege y ayuda tanto emocional como intelectualmente; pero también como algo que brinda seguridad para proyectar el futuro, porque siempre estará ahí para servir de ancla en la conquista de la felicidad. Sin embargo, es pertinente señalar que la familia también puede malograr la felicidad. Una relación familiar conflictiva contribuye al deterioro de su alegría. Así, estas personas adolescentes van aprendiendo que la felicidad se vive conforme se encuentran condiciones felicitarias afectivas, siendo la familia un elemento básico de ello: “... el amor de los padres es aún más poderoso, pero en los mejores casos el sentimiento parental es consecuencia del amor entre los padres" (Russell, 2003, p. 27). Siguiendo a Russell, se puede argumentar que el amor de los padres y madres, y el amor entre ellos, es uno de los sentimientos más poderosos en la conquista de la felicidad, sobre todo en la infancia y la adolescencia.

El otro lazo afectivo es la amistad. La relación con los pares atraviesa su modo de actuar y esa experiencia se acompaña del reconocimiento de 
los compañeros y compañeras, de su aprecio y de su apoyo: "Sin amigos nadie escogería vivir, aunque tuviese los bienes restantes. Los ricos mismos, y las personas constituidas en mando y dignidad, parecen más que todo tener necesidad de amigos" (Aristóteles, 1094a - trad. en 2012, p. 182). Los compañeros y compañeras juegan un papel fundamental en las experiencias felicitarias. Alrededor de ellos y ellas, de algún modo, los estudiantes y las estudiantes basan su felicidad, saben que no pueden tener una vida plena si pierden de vista a los demás: "El secreto de la felicidad es este: que tus intereses sean lo más amplios posible y que tus reacciones a las cosas y personas que te interesan sean, en la medida de lo posible, amistosas y no hostiles" (Russell, 2003, p. 95). Dada la fragilidad de la vida, nuestra felicidad pende de las personas que tenemos, que amamos y estimamos; en este caso concreto, de los amigos y amigas. La amistad, como se infiere de la postura de nuestros sujetos interlocutores, es algo necesaria, pero no sólo eso sino también, como dice Aristóteles (1094a - trad. en 2012), es algo hermoso en la vida del ser humano. Los lazos afectuosos con los amigos y amigas constituyen, como se ha visto, uno de los bienes exteriores más preciados por estos individuos adolescentes. Con los amigos y amigas se llega a establecer lazos de afinidad en ocasiones muy profundos porque, por un lado, complementan lo que en lo individual no pueden tener $\mathrm{y}$, por otro lado, favorecen la configuración de su propia personalidad; es decir, se da un afecto recíproco donde el vínculo de amistad se establece por lo que es cada uno de los amigos y amigas. En este orden de ideas debemos aclarar algo que es esencial, y lo hacemos a partir de las reflexiones que Aristóteles (1094a - 2012) hace sobre el tipo de amistad que cultivan las personas jóvenes. A esta edad, dice el filósofo, la amistad se da preferentemente por la búsqueda de placer, esto es, por los momentos agradables que un amigo o amiga nos hace pasar: "La amistad de los jóvenes parece tener por motivo el placer. Los jóvenes, en efecto, viven por la pasión, y van sobre todo tras lo placentero para ellos y lo presente; pero mudándose de edad, otros deleites sobrevienen" (Aristóteles, 1094a - trad. en 2012, p. 186). Con base en esto es importante acotar que el compañerismo en la escuela no necesariamente constituye la amistad por excelencia, pero sí viene a ser una forma de cultivar y disfrutar cotidianamente la compañía de los pares y, al ser así, se convierte en un vector importante en la consecución diaria de su felicidad. En síntesis, se puede argumentar que nuestros adolescentes tienen su propia forma de apreciar el valor de la amistad, pero justamente este tipo de valoración permite darnos cuenta cuánto vale como fuente de una vida plena.

Seguidamente, es importante analizar que la felicidad también tiene relación con lo que una persona representa para los demás, aunque en nuestros estudiantes este dominio no cuenta con el peso suficiente en su vida. En esta edad, como se observa, no tiene mucho sentido lo que representa tener fama o poder. La admiración pública como necesidad de la vanidad individual -idea postulada por Arendt (1991)-, no es relevante en nuestros sujetos interlocutores. Lo importante, en todo caso, como ellos lo expresan, es contar con el reconocimiento de los amigos y amigas, lo cual nos vuelve a remitir a la importancia de la amistad. Lo que piensan los demás se cierra al círculo de los amigos y amigas. Por tanto, el argumento de que la felicidad también se basa en la consecución de fama y poder no tiene fuerza en los juicios adolescentes. Esta valoración se puede deber, quizás, a la transparencia de su corta vida. La lección a promover en todo caso, es que nuestros individuos adolescentes deben aprender a valorarse a sí mismos y no hacerlo desde la mirada de los demás. Creemos que ellas y ellos ubican de manera precisa que un plan de una vida feliz se afianza en los atributos personales, en lo que tienen y en lo que son, así como en la posesión de los afectos. El poder sobre los demás, los aplausos y los cargos políticos, en los cuales reside en muchas ocasiones la felicidad, no pueden reemplazar tan fácilmente los argumentos esgrimidos en favor de la felicidad. Una cita de Aristóteles (1094a - trad. en 2012, p. 6) es pertinente para sintetizar esta reflexión:

El honor, sin embargo, parece ser un bien harto superficial para ser el que buscamos nosotros, pues manifiestamente está más en quien da la honra que en el que 
la recibe, en tanto que, según podemos presentir desde ahora, el verdadero bien debe ser algo propio y difícil de arrancar de su sujeto.

\section{Conclusiones}

Después de analizar las valoraciones de las personas adolescentes, podemos concluir que existe el consenso de que estar sano, ser alegres, tener familia y amigos y amigas son bienes deseables en sí mismos para tener una vida feliz, y son fuente determinante de experiencias privilegiadas. Pero además, conviene subrayar que cuando se valora de ese modo es una garantía de que la felicidad es compartida. Es preciso recordar que de la lista de dominios que ellas y ellos mencionaron, todos tienen algún sentido en su vida dichosa, pero no todos tienen el mismo énfasis ni la misma intensidad. ¿Cuáles son las razones de ello? Una clasificación de Aristóteles (trad. en 2012) puede ser útil en este razonamiento final. El filósofo clasifica los bienes en tres tipos: exteriores, del alma y del cuerpo. Los del alma son los bienes de máxima propiedad porque se vinculan con el estado interno del ser humano. De esta forma, cuando los estudiantes se representan la felicidad relacionada con la vida afectiva, podemos descubrir que le atribuyen valor a los bienes que satisfacen una necesidad interna relacionada con el afecto. Principalmente son las relaciones con los demás, las maneras de tratarse unos a otros, los que fundamentan los fines de la vida (Berlin, 2009, p. 3). Los bienes exteriores o materiales que constituyen solo una parte complementaria en las trayectorias de las personas, son valorados como menos necesarios en la calificación de una vida dichosa.

Los resultados también explican que las valoraciones acerca de la felicidad se comprenden en cierto modo por los límites históricos y personales en los cuales se encuentran los agentes, esto es, por su contexto; lo que significa que la vida feliz no solo es un asunto meramente conceptual como bien lo establece Domingo (1994), sino que está anclada al mundo cotidiano. Esta reflexión es importante, porque nos invita a pensar en las condiciones materiales, educativas y de esparcimiento deseables para cultivarla no solamente en este sector de la población, sino también en edades como la infancia y la juventud. En cada etapa de la vida, suponiéndolas diversas y distintas, la felicidad tiene sus propias formas de instalarse; por ello se puede afirmar que tiene su particular modo de ser en la adolescencia: se emplaza con sus propios encuentros y desenlaces. En modo alguno se puede pensar que al ser así, la felicidad sea vista por ellos como algo superficial; por el contrario, está relacionada con su desarrollo pleno. Por estas razones, la aspiración a la felicidad, en estos momentos en que están construyendo su plan de vida, no debe ser considerada un ideal lejano y abstracto; por el contrario, los encuentros con los sujetos entrevistados ilustran que esto es posible, y la escuela y la familia pueden ser importantes promotoras en su consecución.

En stricto sensu, se podría objetar que se puede enseñar a las personas adolescentes a ser felices, lo cual tendría sentido pues su encuentro está en el diario vivir, experimentándose de manera íntima y personal; pero sí se puede decir que para vivirla deben aprender a conquistarla para que se instale justificada y permanentemente. Esta es sin duda una pretensión que se puede impulsar en nuestros adolescentes. Ellas y ellos deben aprender a estar en la vida sin excesos, siempre con la mirada puesta en los demás y en las cosas que los rodean; de ahí la importancia de hacer las mediaciones necesarias de temas tan cruciales como éstos, justo en la edad en que se encuentran nuestros interlocutores e interlocutoras. De esta idea podemos inferir que el modo de ser, desde la niñez y la adolescencia, marca el carácter de las personas; por eso la importancia de promover en ellas su merecimiento. Finalmente, podemos destacar que las valoraciones de los individuos entrevistados acerca de los principales dominios sobre la felicidad no presentan diferencias significativas entre los géneros, ni el tipo de establecimiento educativo condicionó la valoración obtenida; esto quiere decir que a pesar de su corta edad y del contexto urbano, los sujetos adolescentes consultados tienen un consenso sobre lo que entraña el significado de la felicidad. Esto no significa que hemos llegado a conclusiones determinantes, más 
bien los resultados invitan a pensar en la pertinencia de una muestra más amplia de población, considerando no solo los individuos adolescentes insertos en el sistema educativo, sino aquellos grupos que. en nuestro medio social, por diversas razones no están en la escuela.

\section{Lista de referencias}

Arendt, H. (1991). La condición humana. Barcelona: Paidós.

Aristóteles (1094a - trad. en 2012). Ética Nicomaquea. $3^{\mathrm{a}}$ ed. México, D. F.: Unam.

Berlin, I. (2009). El estudio adecuado de la humanidad. Antología de ensayos. México, D. F.: Fondo de Cultura Económica.

Brisson, L. (2011). La felicidad según Platón y la tradición platónica. Ser feliz es asimilable al dios. En C. Trueba (coord.) La Felicidad, (pp. 23-31). México, D. F.: UAM, Siglo XXI.

Bruckner, P. (2008). La euforia perpetua. México, D. F.: Tusquets.

Diener, E. (2013). ¿Por qué las sociedades necesitan la felicidad y cuentas nacionales de bienestar? En F. Manzanilla (dir.) 2012 Ranking de la felicidad en México, (pp. 1431). México, D. F.: Universidad Popular Autónoma del Estado de Puebla.

Domingo, A. (1994). Felicidad. En A. Cortina (dir.) 10 palabras claves en ética, (pp. 101153). Navarra: Verbo Divino.

Foot, P. (2002). Bondad Natural. Una visión naturalista de la ética. Barcelona: Paidós.

Hernández, R. Fernández, C. \& Baptista, P. (2014). Metodología de la investigación. México, D. F.: McGraw-Hill.

Loewe, D. (2011). La felicidad y el bienestar subjetivo. En C. Trueba (coord.) La Felicidad, (pp. 362-388). México, D. F.: Universidad Autónoma de México, Siglo XXI.

Marías, J. (1989). La felicidad humana. Madrid: Alianza Editorial.

Nussbaum, M. (2004). La fragilidad del bien. Madrid: La balsa de la Medusa.

Platón (271a-307c - trad. en 1983). Diálogos. Eutidemo. Madrid: Gredos.

Rodríguez, M. J. (2015). Representación social de la noción de felicidad en estudiantes y profesionales de educación y salud de Chile e Italia. Universitas Psychologica 14 (1), 15-29. Doi: 10.11144/Javeriana. upsy141.rsnf.

Rojas, M. (2013). Hacia una sociedad más feliz. En F. Manzanilla (dir.) 2012, Rankig de felicidad en México, (pp. 222-238). México, D. F.: Universidad Popular Autónoma del Estado de Puebla.

Romney, A. K., Weller, S. C. \& Batchelder, W. H. (1986). Culture as consensus. A theory of culture and informant accuracy. American Anthropologist 81 (2), pp. 313338.

Russell, B. (2003). La conquista de la felicidad. Barcelona: De Bolsillo.

Salazar, J. J. \& Arenas, L. (2016). Un modelo económico de felicidad y su evidencia en México. Estudios Sociales: Revista de investigación cientifica, 47 (24), pp. 3-31.

Schopenhauer, A. (2008). El arte del buen vivir. Madrid: Edaf.

Singer, P. (1995). Ética práctica. Cambridge: Cambridge University Press.

Yuni, J. A. \& Urbano, C. (2014). Técnicas para investigar. Buenos Aires: Brujas.

Zagal, H. (2013). Felicidad, placer y virtud. La vida buena según Aristóteles. México, D. F.: Ariel. 